\title{
Prevalence of Asymptomatic Malaria Parasitemia in Odisha, India: A Challenge to Malaria Elimination
}

\author{
Preeti Kumari, ${ }^{1,2}$ Swati Sinha, ${ }^{1,2}$ Renuka Gahtori, ${ }^{1,2}$ Chander Prakash Yadav, ${ }^{1}$ Madan Mohan Pradhan, ${ }^{3}$ Manju Rahi, ${ }^{4}$ \\ Veena Pande, ${ }^{2}$ and Anupkumar R. Anvikar ${ }^{1 *}$ \\ ${ }^{1}$ ICMR - National Institute of Malaria Research, New Delhi, India; ${ }^{2}$ Kumaun University, Nainital, India; ${ }^{3}$ National Vector Borne Disease Control \\ Programme, Odisha, India; ${ }^{4}$ Indian Council of Medical Research, New Delhi, India
}

\begin{abstract}
The prevalence of malaria in India is decreasing, but it remains a major concern for public health administration. The role of submicroscopic malaria and asymptomatic malaria parasitemia and their persistence is being explored. A cross-sectional survey was conducted in the Kandhamal district of Odisha (India) during May-June 2017. Blood samples were collected from 1897 individuals for screening of asymptomatic parasitemia. Samples were screened using rapid diagnostic tests (RDTs) and examined microscopically for Plasmodium species. Approximately $30 \%$ of randomly selected samples $(n=586)$ were analyzed using real-time PCR (qPCR), and the genetic diversity of Plasmodium falciparum was analyzed. The prevalence of Plasmodium species among asymptomatic individuals detected using qPCR was $18 \%$, which was significantly higher than that detected by microscopy examination (5.5\%) or RDT (7.3\%). Of these, $37 \%$ had submicroscopic malaria. The species-specific prevalence among asymptomatic malaria-positive cases for P. falciparum, Plasmodium vivax, and mixed infection (P. falciparum and $P$. vivax) by qPCR was $57 \%, 29 \%$, and $14 \%$, respectively. The multiplicity of infection was 1.6 and 1.2 for the merozoite surface protein-1 gene $(m s p 1)$ and $(m s p 2)$, respectively. Expected heterozygosity was 0.64 and 0.47 for $m s p 1$ and $m s p 2$, respectively. A significant proportion of the study population, 105/586 (18\%), was found to be a reservoir for malaria infection, and identification of this group will help in the development of elimination strategies.
\end{abstract}

\section{INTRODUCTION}

In India, the National Vector Borne Disease Control Programme (NVBDCP) has intensified malaria elimination activities considerably. India estimated 0.39 million malaria cases and 85 deaths in $2018 .{ }^{1}$ India is highly diversified in terms of the distribution of malaria cases. Odisha, an eastern state, has a high prevalence of malaria transmission, contributing to $16.6 \%$ malaria cases and $4.7 \%$ deaths of the total malaria burden in India. ${ }^{2}$

"Asymptomatic malaria infection" refers to malarial parasitemia of any density in a patient who does not have symptoms of fever or acute illness, and who has not received antimalarial treatment in the past few days. ${ }^{3,4}$ Such patients become a "reservoir" for parasite transmission and may act as a precursor of symptomatic malaria. Asymptomatic parasitemia is mostly overlooked during malaria surveillance conducted by the NVBDCP. In addition, obtaining data on malaria from the private health sector is difficult, which could result in underestimation of the prevalence. ${ }^{5}$ The role of asymptomatic parasite carriers in the persistent transmission of malaria in the Indian population is not well understood.

Genetic profiling has shown that a single host coinfected by more than one clone of a malaria-causing parasite is a useful marker of transmission intensity, and also helps in determining the immune response of the human host. ${ }^{6}$ The presence of several clones or multiplicity of infection (MOI) has shown a positive association with the development of an immune response against the mild clinical manifestations of malaria in some circumstances. ${ }^{7}$ Infection from different parasite variants can be a probable cause for the development of immunity against the parasite variants and in a human host may reflect acquired immunity and premonition. ${ }^{8}$ Different parameters

* Address correspondence to Anupkumar R. Anvikar, ICMR-National Institute of Malaria Research, Dwarka Sector 8, New Delhi, India 110077. E-mail: anvikar@gmail.com (e.g., parasite variants and infection duration) can give rise to clonal antigenic variations as well as allelic polymorphism.

Despite control measures, malaria transmission persists in India. Recent studies have shown that submicroscopic, asymptomatic infection in Plasmodium falciparum and Plasmodium vivax is very important, but very few studies have reported such infection in an Indian context. ${ }^{8,11}$ In India, elimination strategies need to be calibrated precisely according to the endemicity in the state to understand the true disease burden, including that asymptomatic and submicroscopic parasite densities are missed during the routine diagnosis. Therefore, this study aimed to estimate the prevalence of parasite infection among asymptomatic individuals.

\section{METHODS}

Survey site and sample collection. A cross-sectional mass survey was conducted during the pre-monsoon season in malaria hyperendemic district Kandhamal (coordinates: $20.47^{\circ} \mathrm{N} 84.23^{\circ} \mathrm{E}, 83.30^{\circ} \mathrm{E}$ to $84-48^{\circ} \mathrm{E}$ longitude, and $19-34^{\circ} \mathrm{N}$ to $20-54^{\circ}$ latitude) in Odisha, India. Kandhamal district has 2,515 villages, with an estimated population of $\sim 733,110$. It receives rain mainly during the southwest monsoon season (June to September) and some rainfall during the northeast monsoon (December to January) ${ }^{8,10}$ Kandhamal district is surrounded by mountains and semi-forests located at an altitude of $700 \mathrm{~m}$ above sea level, with an average annual rainfall of $1,522.95 \mathrm{~mm}$. The study site was based on the hyperendemicity of malaria, tribal population.

The mass survey was conducted in 13 villages of Kandhamal district; convenience sampling was used. Individuals available at the sample collection site during the time of mass survey (0900-1600 hours), who agreed to participate in our study, were asked questions on malaria history, intake of antimalarials, and other illness necessitating a visit to a medical facility, and were screened by bivalent malaria rapid diagnostic tests (RDTs). 


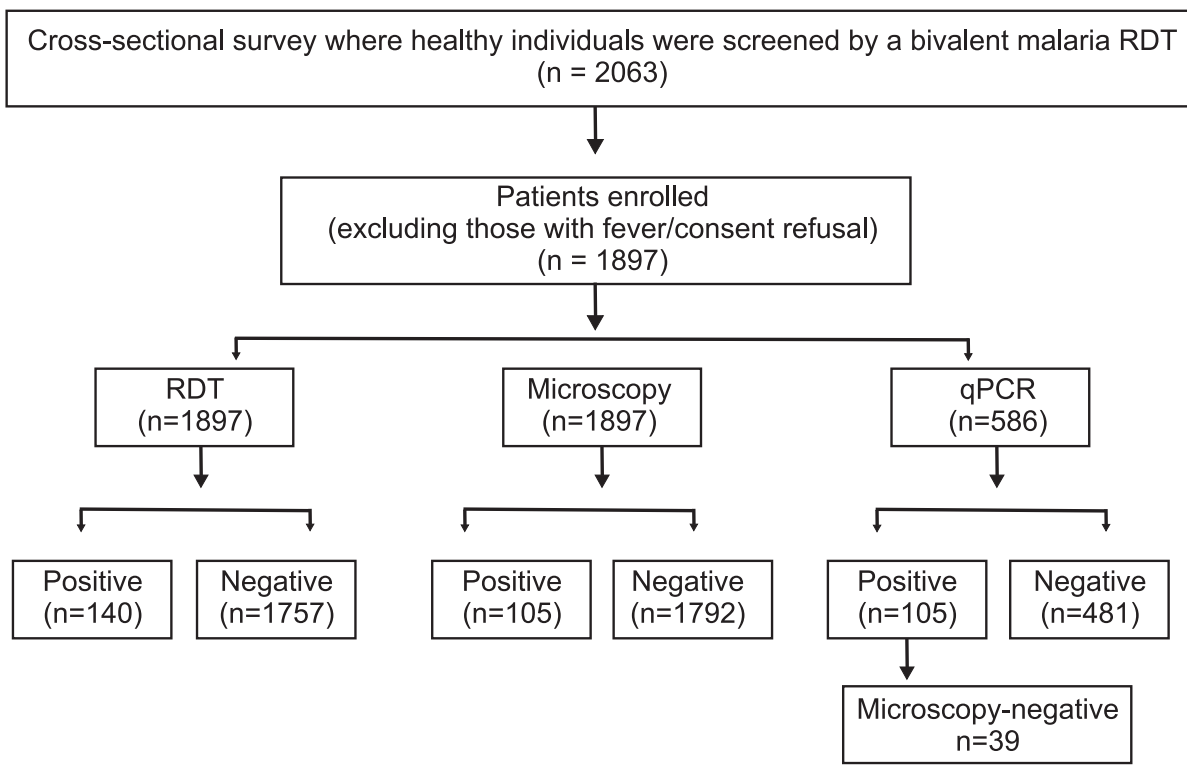

FIGURE 1. Flowchart illustrating the sample size, diagnostic procedures, and prevalence of parasite infection among asymptomatic individuals. $\mathrm{qPCR}=$ real-time PCR; RDT = rapid diagnostic test.

During the survey, 2,063 individuals aged 1-85 years participated. They were classified into three categories irrespective of gender: 1 to $>5$ years, 5 to $>18$ years, 18 years and above. All individuals who were malaria positive according to RDTs were provided with first-line antimalarial drugs ( $P$. falciparum: artesunate + sulfadoxine - pyrimethamine + primaquine; $P$. vivax: chloroquine + primaquine) according to guidelines set by the Indian government. Individuals with malaria symptoms, a recent history of malaria, or necessitating consultation at a medical facility during the day of sample collection were excluded from the study.

After fulfilling the inclusion and exclusion criteria, 1,897 individuals from 13 villages were enrolled in the present study. We did not instigate follow-up for missing individuals or patients who were malaria positive according to an RDT. Blood smears were prepared on microscope slides, air-dried, and stored in slide boxes for staining. Blood spots were prepared from finger-pricks on 3-mm filter paper (Whatman, Maidstone, United Kingdom), air-dried, and stored in a separate zip pouch with a desiccant at $4^{\circ} \mathrm{C}$ following appropriate laboratory practice instructions. Samples were transported to the Indian Council of Medical Research-National Institute of Malaria Research (ICMR-NIMR), New Delhi, for molecular studies.

Case definition for asymptomatic and submicroscopic malaria parasitemia. To define the clinical status for the identification of true asymptomatic malaria parasitemia at the time of the survey, individuals with fever (with or without parasites) ongoing for 1 week before the survey were excluded from the study. Because of the prophylactic effect of sulfadoxine/pyrimethamine, ${ }^{11}$ individuals treated within 4 weeks before the survey were also excluded from the asymptomatic group. All individuals without a fever and malaria positive (presence of parasitemia) or positive according to RDTs, microscopy, or real-time PCR (qPCR) were considered to have asymptomatic malaria parasitemia. Patients who were microscopy negative with the presence of Plasmodium DNA in the molecular diagnosis were defined as being infected with a submicroscopic malaria-causing parasite. Patients who were malaria negative by microscopy and qPCR but malaria positive by an RDT were considered to be false-positive cases.

Sample analysis by microscopy. Microscopic examination was performed by the WHO-trained ICMR-NIMR staff. Thick and thin smears were prepared from samples of fingerprick blood, stained with $10 \%$ Giemsa solution, and examined for $>200$ leukocytes for a thick film and > 200 microscopic fields with a $\times 100$ objective. As a standard protocol for microscopy, double-reading was carried out for all malariapositive slides and $10 \%$ of randomly selected malarianegative slides by independent microscopists to monitor examination quality. The microscopist who examined the corresponding blood slides of participants was blinded to the RDT results.

DNA isolation and qPCR. Genomic DNA was extracted for a randomly selected subset of 586 samples. Three punches of blood spots collected on a filter paper (Whatman) were taken from each sample and used for DNA isolation with genomic DNA extraction kit, following the manufacturer's (Qiagen, Stanford, VA) recommendations. A malaria-causing parasite was identified by a species-specific probe based on qPCR targeting a conserved 18S rRNA gene of $P$. falciparum and $P$. vivax as described by Kamau et al. ${ }^{12}$ Amplification was carried out using a "reaction cocktail" containing $2 \mu \mathrm{L}$ of template DNA and $1 \times$ QuantiFast $^{\mathrm{TM}}$ Probe PCR Master Mix without ROX dye (Qiagen), $0.2 \mu \mathrm{M}$ of each probe, and $0.4 \mu \mathrm{M}$ of each primer. Real-time PCR assays were carried out with

\section{TABLE 1}

Distribution of age among the total samples diagnosed by microscopy, a rapid diagnostic test, and randomly selected subset of samples for real-time PCR

\begin{tabular}{ccc}
\hline Age category (years) & $\begin{array}{c}\text { In total sample } \\
(N=1,897), n(\%)\end{array}$ & $\begin{array}{c}\text { In } 30 \% \text { of a randomly selected } \\
\text { sample }(N=586), n(\%)\end{array}$ \\
\hline$<5$ & $134(7.0)$ & $49(8.4)$ \\
$5-17$ & $684(36.1)$ & $232(39.6)$ \\
$\geq 18$ & $1,079(56.9)$ & $305(52.0)$ \\
\hline
\end{tabular}



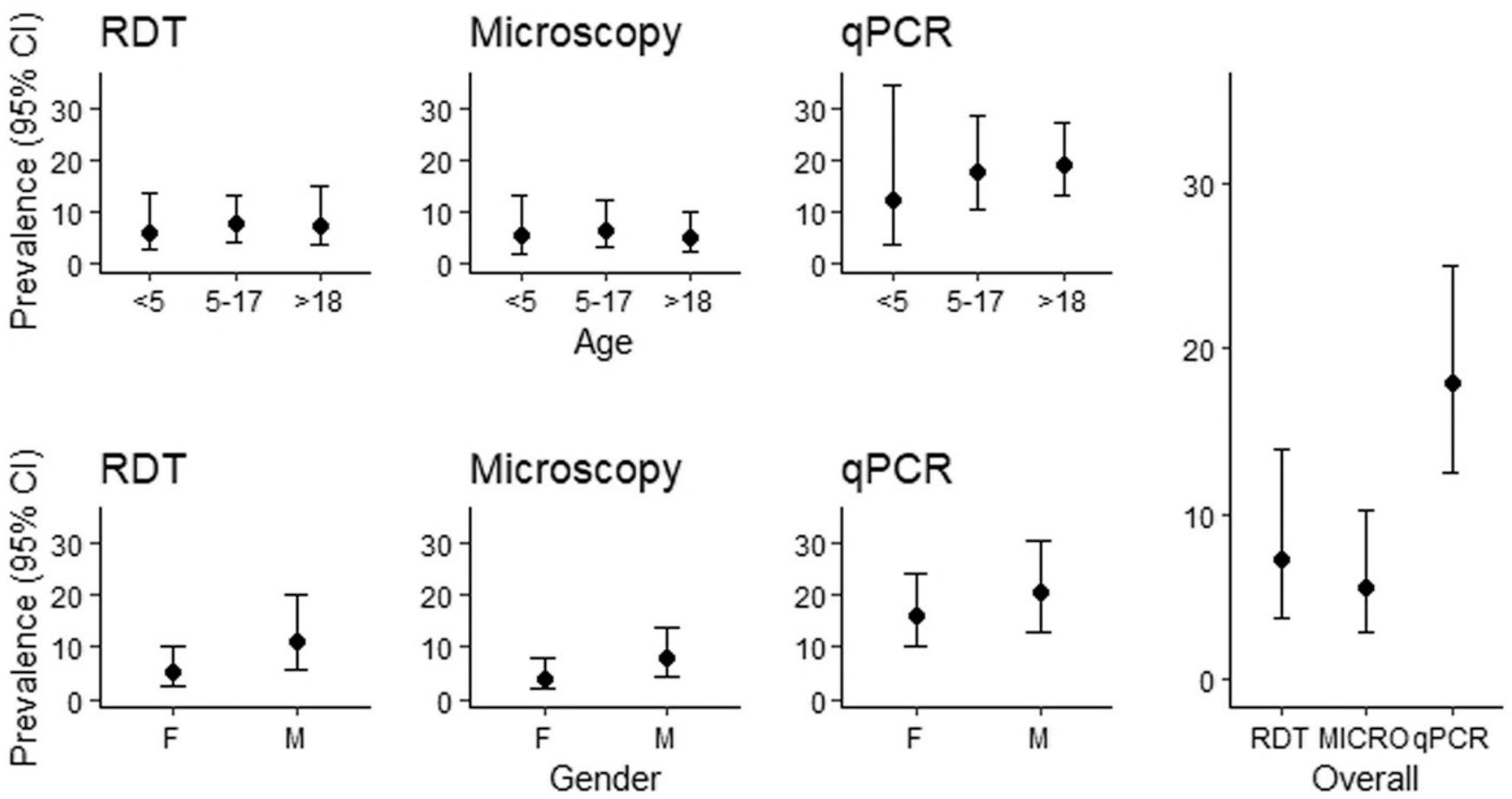

FIGURE 2. Prevalence of parasite infection among the asymptomatic population detected by a rapid diagnostic test (RDT), microscopy, and realtime PCR (qPCR).

specific controls, including the non-template control. Samples were tested in triplicate. The thermal profile used for qPCR was 5 minutes at $95^{\circ} \mathrm{C}, 3$ seconds at $95^{\circ} \mathrm{C}$, and 30 seconds at $60^{\circ} \mathrm{C}$ for 40 cycles in a LC480 LightCycler (Roche, Basel, Switzerland).

Genotyping. Genotyping of $P$. falciparum-positive samples obtained by qPCR was conducted on two highly polymorphic regions of the $P$. falciparum genome: $m s p 1$ and $m s p 2$. PCR amplification of $m s p 1$ and $m s p 2$ comprised an initial denaturation at $95^{\circ} \mathrm{C}$ for 5 minutes followed by 30 cycles of $95^{\circ} \mathrm{C}$ for 1 minute, $58^{\circ} \mathrm{C}$ for 2 minutes, $72^{\circ} \mathrm{C}$ for 2 minutes, and a final extension of $72^{\circ} \mathrm{C}$ for 5 minutes. The amplification parameters for nested PCR of msp1 (MaD20, K1, and R033) alleles and msp2 (FC27 and 3D7) alleles were identical to those of the primary cycle, except for the annealing temperature, which was $61^{\circ} \mathrm{C}$ for 2 minutes. PCRs were carried out with a positive control of specific alleles along with a negative control. The PCR products for $m s p 1$ and $m s p 2$ were visualized on $1.5 \%$ ethidium bromide-stained agarose gels under ultraviolet illumination.

Multiplicity of infection and heterozygosity index. The $\mathrm{MOI}$ was calculated as the proportion of the total number of $P$. falciparum genotypes for each marker divided by the number of positive PCR isolates. Expected heterozygosity $\left(H_{\mathrm{E}}\right)$ is defined as the probability of being infected by two parasites with different alleles at a given locus. It is calculated using the following formula: $H_{\mathrm{E}}=[n /(n-1)]\left[1-\sum \mathrm{pi}^{2}\right]$, where $n$ is the total number of isolates and pi is the allele frequency at a given locus.

Statistical analysis. Categorical variables are presented as the number (\%). The association of various factors with malaria positivity was assessed using logistic regression and is presented as odds ratios (ORs). Data analyses were carried out using R v3.4 (R Center for Statistical Computing, Vienna, Austria) and Stata 15.0 (www.Stata.com).

Ethical approval of the study protocol. This study was carried out at the ICMR-NIMR in New Delhi, India. The study protocol was approved by the Ethics Committee of ICMRNIMR, who waived the requirement for written informed consent.

\section{RESULTS}

Demographic characteristics of the study population. The mass survey was conducted in 13 hyperendemic villages of Kandhamal district, and 2,063 individuals were screened. A total of 166 patients carrying malaria symptoms were excluded from the study, and the remaining 1,897 individuals with no malaria symptoms or with no recent history of malaria were enrolled. Most of the participants enrolled in our study were female $(61 \%)$. The median age of study participants was 28 years. All samples were screened using an RDT and microscopy, and only a random subset of 586 participants (30\%), irrespective of age, were screened using qPCR (Figure 1). The distribution of all three age-groups is comparable with the complete study population of 1,897 slides (Table 1).

TABLE 2

Validation of an RDT to detect parasites using qPCR

\begin{tabular}{llcccr}
\hline & \multicolumn{4}{c}{ qPCR } \\
\cline { 3 - 6 } & & Negative & Positive & qPCR not performed & Total \\
\hline RDT & Negative & 473 & 47 & 1,237 & 1,757 \\
& Positive & 8 & 58 & 74 & 140 \\
& Total & 481 & 105 & 1,311 & 1,897 \\
\hline \multicolumn{2}{l}{ qPCR = real-time PCR; RDT $=$ rapid diagnostic test. }
\end{tabular}


TABLE 3

Validation of microscopy to detect parasites using $\mathrm{QPCR}$

\begin{tabular}{llrrcr}
\hline & & \multicolumn{3}{c}{ qPCR } \\
\cline { 3 - 6 } & & Negative & Positive & qPCR not performed & Total \\
\hline Microscopy & Negative & 478 & 50 & 1,264 & 1,792 \\
& Positive & 3 & 55 & 47 & 105 \\
& Total & 481 & 105 & 1,311 & 1,897 \\
\hline qPCR = real-time PCR. & & & &
\end{tabular}

Prevalence of parasite infection among the asymptomatic population. Parasite infection prevalence among the asymptomatic population was $7.4 \%, 5.5 \%$, and $18 \%$ diagnosed using RDT, microscopy, and $\mathrm{qPCR}$, respectively (Figure 2). A higher prevalence of asymptomatic parasitemia was observed for males than for females across all three diagnostic methods. There was no difference in the prevalence of parasite infection among asymptomatic individuals across three age-groups when the diagnosis was made using RDT and microscopy. Testing by qPCR showed a higher prevalence in adults aged $\geq 18$ years (19\%) and young children aged $5-17$ years $(18 \%$ compared with that in children aged $<5$ years $(12.2 \%)$. The prevalence of parasitemia among parasitemic asymptomatic individuals in study villages varied from $0 \%$ to $35.7 \%$ when diagnosed with RDT, 1.2-21.4\% when diagnosed with microscopy, and 3.5-75\% when diagnosed with $\mathrm{GPCR}$. Rapid diagnostic test results were compared with qPCR when eight individuals were found to be RDT positive and qPCR negative for $P$. falciparum, whereas, when microcopy results were compared with $\mathrm{QPCR}, 50$ individuals were microscopy negative and qPCR positive. However, of these
50 individuals, 11 were RDT positive, so we excluded these 11 and rechecked the remaining 39 samples by microscopy and qPCR; results remained microscopy negative and qPCR positive. Hence, submicroscopic parasitemia (microscopy negative but qPCR positive) was confirmed (Tables 2 and 3).

Asymptomatic malaria infection and its characteristics. The association of gender and age with asymptomatic malaria was assessed using logistic regression. Males were more prone to asymptomatic malaria if diagnosed using an RDT (10\%; OR, 2.31) and microscopy (7.8\%; OR, 1.9). However, an association was not observed between gender and asymptomatic malaria if diagnosis was made using qPCR (20.5\%; OR, 1.3). Age was not associated with asymptomatic parasitemia across the three diagnostic methods (Table 4).

Prevalence of infection with Plasmodium species. Species distribution among parasitemic asymptomatic individuals included a majority of $P$. falciparum monoinfections detected using qPCR (57\%), microscopy (82\%), and RDT $(77 \%)$, followed by $P$. vivax mono-infections by qPCR (29\%), microscopy (15\%), and RDT (19\%). Coinfection of $P$. falciparum and $P$. vivax was found to be $14 \%$ when detected using qPCR, 3\% using microscopy, and $4 \%$ using an RDT (Figure 3). Data obtained from three diagnostic methods had discrepancy in 14 samples such as 11 samples were qPCR positive, RDT positive but microscopy negative and three samples were $P$. vivax by qPCR and microscopy but mixed ( $P$. falciparum and $P$. vivax) by RDT. We used qPCR as the "gold standard" to determine sensitivity and specificity of $63 \%$ and $99 \%$, respectively, for microscopy,

TABLE 4

Association of age and gender in asymptomatic malaria by an RDT, microscopy, and qPCR

\begin{tabular}{|c|c|c|c|}
\hline \multicolumn{4}{|c|}{ RDT } \\
\hline & Negative $(n=1,757), n(\%)$ & Positive $(n=140), n(\%)$ & Odds ratio \\
\hline \multicolumn{4}{|l|}{ Gender } \\
\hline Female & $1,094(94.8)$ & $59(5.1)$ & Reference \\
\hline Male & $663(89.1)$ & 81 (10.9) & 2.3 \\
\hline \multicolumn{4}{|l|}{ Age (years) } \\
\hline$<5$ & $126(94.1)$ & $8(5.9)$ & Reference \\
\hline $5-17$ & 631 (92.3) & $53(7.7)$ & 1.3 \\
\hline$\geq 18$ & $1,000(92.7)$ & $79(7.3)$ & 1.2 \\
\hline \multicolumn{4}{|c|}{ Microscopy } \\
\hline & Negative $(n=1,792), n(\%)$ & Positive $(n=105), n(\%)$ & Odds ratio \\
\hline \multicolumn{4}{|l|}{ Gender } \\
\hline Female & $1,106(95.9)$ & $47(4.1)$ & Reference \\
\hline Male & $686(92.2)$ & $58(7.8)$ & 1.9 \\
\hline \multicolumn{4}{|l|}{ Age (years) } \\
\hline$<5$ & $127(94.8)$ & $7(5.2)$ & Reference \\
\hline $5-17$ & 640 (93.6) & $44(6.4)$ & 1.2 \\
\hline$\geq 18$ & $1,025(95)$ & $54(5)$ & 0.95 \\
\hline \multicolumn{4}{|c|}{ qPCR } \\
\hline & Negative $(n=481), n(\%)$ & Positive ( $n=105), n(\%)$ & Odds ratio \\
\hline \multicolumn{4}{|l|}{ Gender } \\
\hline Female & 283 (83.9) & 54 (16.1) & Reference \\
\hline Male & 198 (79.5) & $51(20.5)$ & 1.3 \\
\hline \multicolumn{4}{|l|}{ Age (years) } \\
\hline$<5$ & $43(87.8)$ & $6(12.2)$ & Reference \\
\hline $5-17$ & 191 (82.3) & $41(17.7)$ & 1.5 \\
\hline$\geq 18$ & 247 (80.9) & $58(19.1)$ & 1.7 \\
\hline
\end{tabular}




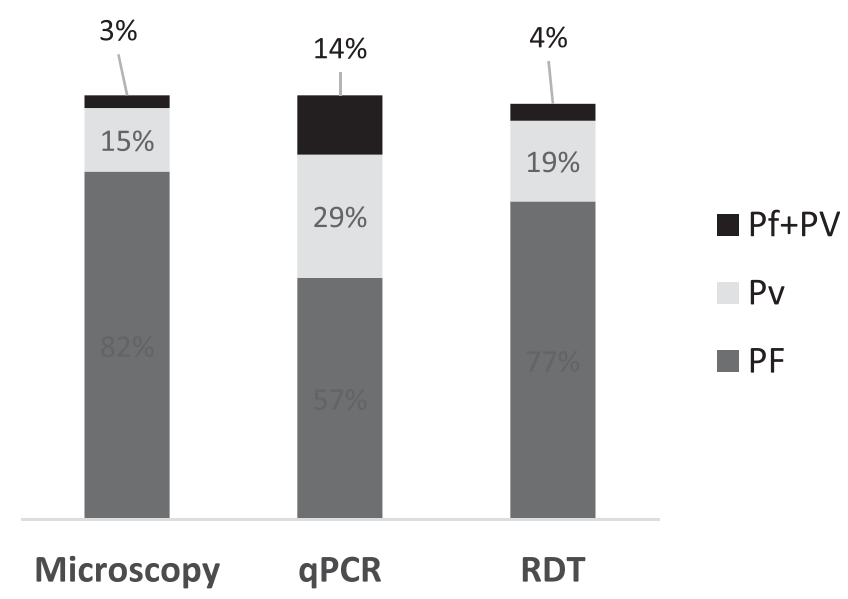

FIGURE 3. Distribution of malaria-causing species among positive cases across the three diagnostic methods. QPCR = real-time PCR; RDT = rapid diagnostic test. This figure appears in color at www.ajtmh.org.

and $55 \%$ and $93 \%$ for RDT, respectively. The positive predictive value and negative predictive value was $96 \%$ and $93 \%$ for microscopy, and $64 \%$ and $91 \%$ for RDT, respectively (Table 5). Among all three age categories, agegroup $>18$ years carried the maximum infection, with most having $P$. falciparum infection $(33 \%)$ followed by $P$. vivax infection (14.2\%) and mixed infection (P. falciparum and P. vivax) (7.6\%).

Allelic diversity of $m s p 1$ and $m s p 2$ of $P$. falciparum isolates. Alleles of $m s p 1$ and $m s p 2$ were categorized according to the size of the PCR amplified product. Successful amplification of the msp1 locus was found in $48 / 60$ $(80 \%)$ isolates where 10 genotypes were identified. In the msp 1 locus, $33 \%, 6 \%$, and $19 \%$ isolates contained a single genotype of Mad20, K1, and Ro33 alleles, respectively. Multiple genotypes of $m s p 1$ were observed in $\mathrm{K} 1+$ Ro33 (12.5\%), K1 + Mad20 (8.3\%), Ro33 + Mad20 (6.3\%), and $\mathrm{K} 1$ + Ro33 + Mad20 (14.6\%) isolates. In the msp2 locus, 57/ $60(95 \%)$ isolates had successful amplification with $13 \mathrm{ge}-$ notypes. For the msp2 locus, a single genotype for FC27 and 3D7 was observed to be in $45.6 \%$ and $28.1 \%$ of isolates, respectively, whereas multiple genotypes for FC27 and 3D7 were observed in $26.3 \%$ isolates. The size of the polymorphism ranged from 120 to $250 \mathrm{bp}$ and 280 to $800 \mathrm{bp}$ in $m s p 1$ and $m s p 2$, respectively. We observed that $33 \%$ of isolates in $m s p 1$ and $28 \%$ in $m s p 2$ were multiclonal, which suggested the presence of two or more alleles. Merozoite surface protein-1 carried more multiclonal infections than $m s p 2$ (Table 6). Merozoite surface protein-1 and $m s p 2$ multiclonal infections carried $70 \%$ and $58 \%$ asymptomatic infections, respectively, and $30 \%$ and $42 \%$ were submicroscopic for $m s p 1$ and $m s p 2$ alleles, respectively. The overall mean $\mathrm{MOI}$ of $P$. falciparum isolates from Odisha was 1.4. The mean $\mathrm{MOI}$ for $m s p 1$ was 1.6 and 1.2 for $m s p 2$ (Table 6). Expected heterozygosity was calculated to assess the discriminatory power of the two individual markers. Expected heterozygosity estimates the segment of all parasites that would be heterozygous for any of the two loci. Expected heterozygosity was 0.64 for K1, Mad20, and Ro33 alleles of $m s p 1$, and 0.47 for FC27 and 3D7 alleles of $m s p 2$.

\section{DISCUSSION}

Odisha is a hyperendemic malaria region that covers almost one quarter of the malaria burden of India. The contributory factors for malaria transmission in this state are hilly forested areas inhabited by tribal populations. Molecular methods used for the diagnosis of malaria-causing parasites play a key part in the identification of submicroscopic and asymptomatic malaria parasitemia.

In this cross-sectional study, we observed a $18 \%$ prevalence of parasite infection among asymptomatic population including P. falciparum (57\%), P. vivax (29\%), and mixed infection (P. falciparum and $P$. vivax) (14\%) and submicroscopic infection (37\%) in Odisha. When RDT results were compared with qPCR data, eight samples were found to be RDT positive and qPCR negative, which may have been a result of histidinerich protein-2 persistence. ${ }^{13}$ When microscopy results were compared with qPCR data, 11 samples were microscopy negative, qPCR positive, and RDT positive (Tables 2 and 3). All 11 samples with this discrepancy were rechecked by microscopy and qPCR, but the result remained the same; these results could have been because of poor quality of smearing and staining.

In India, very few studies have used PCR to identify parasitemia that are submicroscopic and asymptomatic. A study conducted in June 2012 showed that Purulia district (which neighbors Odisha) had a prevalence of asymptomatic $P$. falciparum infection of $8.4 \%$ detected using PCR and $6.8 \%$ using microscopy, whereas the prevalence of submicroscopic infection was $1.5 \% .{ }^{14} \mathrm{~A}$ survey study conducted in Chhattisgarh in India in year 2016 detected a prevalence of sub-patent parasitemia of $10 \%$ and prevalence of asymptomatic parasitemia of $18 \% .{ }^{11}$ In Rourkela district within Odisha, a survey study reported the prevalence of asymptomatic parasitemia to be $8.3 \%$ and the prevalence of submicroscopic cases to be $5.7 \% .{ }^{8}$ Our data are in accordance with the prevalence of highly asymptomatic parasitemia transmission found in Odisha reported by Pradhan et al. ${ }^{15}$ Taken together, these studies suggest that a surveillance study based on symptoms and the sensitivity of RDTs and microscopy in areas with a low density of parasites may lead to underestimation of the number of malaria-causing parasites. Underestimation of the burden of sub-patent and asymptomatic malaria parasitemia can have important implications on malaria surveillance.

We focused on the complexity of asymptomatic malaria parasitemia to understand the molecular epidemiology of the study population. The $\mathrm{MOI}$ is a proven marker of the intensity of malaria transmission in certain studies. ${ }^{16,17}$ However,

TABLE 5

Predictive ability of an RDT and microscopy taking real-time PCR as the gold standard

\begin{tabular}{lccrr}
\hline & Sensitivity, \% (95\% Cl) & Specificity, \% (95\% Cl) & Positive predictive value, \% (95\% Cl) & Negative predictive value, \% (95\% Cl) \\
\hline Microscopy & $63(53-72)$ & $99(98-100)$ & $95.7(88-99)$ & $93(90-95)$ \\
RDT & $55(45-65)$ & $93(91-95)$ & $64(53-74)$ & $91(88-93)$ \\
\hline
\end{tabular}

$\mathrm{RDT}$ = rapid diagnostic test. 
TABLE 6

Genetic diversity of $m s p 1$ and $m s p 2$ of Plasmodium falciparum isolates from Odisha, India

\begin{tabular}{|c|c|c|c|c|c|}
\hline & Allelic family & Frequency, $n(\%)$ & Allele size (bp) & Number of variants & Multiplicity of infection \\
\hline \multirow{7}{*}{$m s p 1(n=48)$} & $\mathrm{K} 1$ & $16(33.3)$ & $180-250$ & 4 & 1.6 \\
\hline & Mad20 & 9 (18.7) & $120-200$ & 3 & \\
\hline & $\mathrm{RO} 33$ & $3(6.3)$ & $120-160$ & 3 & \\
\hline & $\mathrm{K} 1+\mathrm{RO} 33$ & $6(12.5)$ & - & - & \\
\hline & $\mathrm{K} 1+\mathrm{Mad} 20$ & $4(8.3)$ & - & - & \\
\hline & $\mathrm{RO} 33$ + Mad20 & $3(6.3)$ & - & - & \\
\hline & $\mathrm{K} 1+\mathrm{RO} 33+\mathrm{Mad} 20$ & $7(14.6)$ & - & - & \\
\hline \multirow[t]{3}{*}{$m s p 2(n=57)$} & FC27 & $26(45.6)$ & $380-700$ & 6 & 1.2 \\
\hline & 3D7 & $16(28.1)$ & $280-800$ & 7 & \\
\hline & $\mathrm{FC} 27+3 \mathrm{D} 7$ & $15(26.3)$ & - & - & \\
\hline
\end{tabular}

transmission intensity is regulated by various other factors, such as endemicity and vector-biting behavior. ${ }^{18} \mathrm{~K} 1$ and 3D7 alleles were found to be more diverse in msp1 and msp2 blocks, respectively, than other alleles. Age did not correlate with the $\mathrm{MOI}$ in the present study-findings that are similar to those of studies performed in Sudan, Western Kenya, and Nigeria. ${ }^{19,20}$ The development of anti-parasite-specific immunity may relate to the endemicity of the geographic distribution. The within-host diversity of malaria can result in the amplification of the number of $\mathrm{B}$-cell clones due to parasite infection. $^{24}$

To eliminate the foci of asymptomatic carriers, numerous strategies have been suggested that may reduce the parasite burden, ${ }^{25}$ including vector control, intermittent preventive treatment, and periodic population-based cross-sectional surveys in the affected region. Vector control is one of the important strategies followed in malaria control, and is dependent on the understanding of biology, ecology, hostfeeding preference, and genetics of vectors. Methods used for effective control of vectors in India are indoor residual sprays, bed nets, and long-lasting insecticide-treated nets. Whether low-density parasites are transmissible is controversial. ${ }^{9}$ Several studies have shown that submicroscopic $P$. falciparum, as well as $P$. vivax, can be transmitted via mosquitoes. ${ }^{22}$ Identification and treatment of patients with subclinical malaria are strategic tools for countries aiming for malaria elimination. We suggest that more robust and thorough data should be collected using a sensitive method for malaria diagnosis. Real-time PCR is highly sensitive for the diagnosis of sub-patent parasitemia, but is not suitable for test-and-treat approaches in field-based studies because it requires laboratory facilities and skilled molecular biologists, and is expensive. We suggest that a more robust and point-of-care method is required for thorough collection of data. However, the limited sensitivity of RDTs and microscopy should be acknowledged when estimating the true burden of malaria. ${ }^{9}$

Mass drug treatment along with effective control of vectors is recommended to accelerate the interruption of parasite transmission, ${ }^{23}$ but, inevitably, protective immunity will be lost eventually if this effort is successful. ${ }^{24}$ The prevalence of asymptomatic parasitemia and factors affecting parasite transmission vary geographically and may also be influenced by the host, parasite, and environment. ${ }^{25,26}$ Identification of such parameters may help in the elimination program for malaria.

Our study design has faced several limitations. First, it was cross-sectional with limited resources that did not allow for inclusion of symptomatic patients. Further studies should include surveys of symptomatic and asymptomatic patients with malaria. Second, the ratio of females to males was high, which was likely because males were working in forest/farm areas during sample collection. Hence, in future studies, equal numbers of males and females need to be evaluated.

To plan an effective elimination strategy for malaria, the role of asymptomatic parasitemia and its mechanism of transmission as well as its correlation with acquired immunity and malaria strains must be determined in follow-up studies with large sample sizes and a robust study design. A policy change in the diagnosis and treatment of malaria can be of potential benefit in this regard.

Received January 8, 2020. Accepted for publication June 17, 2020.

Published online August 10, 2020.

Acknowledgments: We thank the director in charge, National Institute of Malaria Research, Delhi, India, for providing laboratory facilities. We thank Mritunjay Saxena, the molecular biologist, and field staff at New Delhi and NVBDCP, Odisha, for their help and support in conducting the mass survey in the endemic areas of Odisha, and the Indian Council of Medical Research (ICMR) for providing a grant to carry out this study.

Financial support: This study was supported by a grant (80/999/2015ECD-I) from the Indian Council of Medical Research (ICMR) and by a National Institute of Malaria Research fund.

Authors' addresses: Preeti Kumari, Swati Sinha, Renuka Gahtori, Chander Prakash Yadav, and Anupkumar R. Anvikar, ICMR - National Institute of Malaria Research, New Delhi, India, E-mails: preet.preet165@gmail.com, swati.microbio04@gmail.com, renuka gahtori@rediffmail.com, cpyadav123@gmail.com, and anvikar@gmail. com. Madan Mohan Pradhan, National Vector Borne Disease Control Programme, Odisha, India, E-mail: drmmpradhan@gmail.com. Manju Rahi, Indian Council of Medical Research, New Delhi, India, E-mail: drmanjurahi@gmail.com. Veena Pande, Kumaun University, Nainital, India, E-mail: veena_kumaun@yahoo.co.in.

\section{REFERENCES}

1. Malaria Site, 2018. Incidence of Malaria in India. Available at: https://www.malariasite.com/malaria-india/. Accessed September 15, 2019.

2. Sahu SS, Gunasekaran K, Vanamail P, Jambulingam P, 2013. Persistent foci of falciparum malaria among tribes over two decades in Koraput district of Odisha state, India. Malar J 12: 72.

3. Chen I, Clarke SE, Gosling R, Hamainza B, Killeen G, Magill A, O'Meara W, Price RN, Riley EM, 2016. "Asymptomatic" malaria: a chronic and debilitating infection that should be treated. PLoS Med 13: e1001942.

4. Manjurano $A$, Okell L, Lukindo $T$, Reyburn $H$, Olomi R, Roper $C$, Clark TG, Joseph S, Riley EM, Drakeley C, 2011. Association of sub-microscopic malaria parasite carriage with transmission intensity in north-eastern Tanzania. Malar J 10: 370. 
5. Das A, Anvikar AR, Cator LJ, Dhiman RC, Eapen A, Mishra N, Nagpal BN, Nanda N, Raghavendra K, Read AF, 2012. Malaria in India: the center for the study of complex malaria in India. Acta Trop 121: 267-273.

6. Zhong D, Koepfli C, Cui L, Yan G, 2018. Molecular approaches to determine the multiplicity of Plasmodium infections. Malar J 17: 172.

7. Agyeman-Budu A, Brown C, Adjei G, Adams M, Dosoo D, Dery D, Wilson M, Asante KP, Greenwood B, Owusu-Agyei S, 2013. Trends in multiplicity of Plasmodium falciparum infections among asymptomatic residents in the middle belt of Ghana. Malar J 12: 22.

8. van Eijk AM, Sutton PL, Ramanathapuram L, Sullivan SA, Kanagaraj D, Priya GSL, Ravishankaran S, Asokan A, Sangeetha V, Rao PN, 2019. The burden of submicroscopic and asymptomatic malaria in India revealed from epidemiology studies at three varied transmission sites in India. Sci Rep 9: $1-11$.

9. Okell LC, Bousema T, Griffin JT, Ouédraogo AL, Ghani AC, Drakeley CJ, 2012. Factors determining the occurrence of submicroscopic malaria infections and their relevance for control. Nat Commun 3: 1-9.

10. Attri S, Tyagi A, 2010. Climate Profile of India. New Delhi, India: Environment Monitoring and Research Center, India Meteorology Department.

11. Chourasia M, Raghavendra K, Bhatt $R$, Swain $D$, Valecha $N$, Kleinschmidt I, 2017. Burden of asymptomatic malaria among a tribal population in a forested village of central India: a hidden challenge for malaria control in India. Public Health 147: 92-97.

12. Kamau E, Alemayehu S, Feghali KC, Saunders D, Ockenhouse CF, 2013. Multiplex qPCR for detection and absolute quantification of malaria. PLoS One 8: e71539.

13. Iqbal J, Siddique A, Jameel M, Hira PR, 2004. Persistent histidinerich protein 2, parasite lactate dehydrogenase, and panmalarial antigen reactivity after clearance of Plasmodium falciparum monoinfection. J Clin Microbiol 42: 4237-4241.

14. Ganguly S, Saha P, Guha SK, Biswas A, Das S, Kundu PK, Maji AK, 2013. High prevalence of asymptomatic malaria in a tribal population in eastern India. J Clin Microbiol 51: 1439-1444.

15. Pradhan S, Pradhan MM, Dutta A, Shah NK, Joshi PL, Pradhan K, Sharma S, Daumerie PG, Banerji J, Duparc S, 2019. Improved access to early diagnosis and complete treatment of malaria in Odisha, India. PLoS One 14: e0208943.
16. Koepfli C, Ross A, Kiniboro B, Smith TA, Zimmerman PA, Siba P, Mueller I, Felger I, 2011. Multiplicity and diversity of Plasmodium vivax infections in a highly endemic region in Papua New Guinea. PLoS Negl Trop Dis 5: e1424.

17. Barry AE, Schultz L, Senn N, Nale J, Kiniboro B, Siba PM, Mueller I, Reeder JC, 2013. High levels of genetic diversity of Plasmodium falciparum populations in Papua New Guinea despite variable infection prevalence. Am J Trop Med Hyg 88: 718-725.

18. Mohammed H, Kassa M, Mekete K, Assefa A, Taye G, Commons RJ, 2018. Genetic diversity of the msp-1, msp-2, and glurp genes of Plasmodium falciparum isolates in northwest Ethiopia. Malar J 17: 386.

19. Bakhiet AM, Abdel-Muhsin A-MA, Elzaki S-EG, Al-Hashami Z, Albarwani HS, AIQamashoui BA, Al-Hamidhi S, Idris MA, Elagib AA, Beja-Pereira A, 2015. Plasmodium falciparum population structure in Sudan post artemisinin-based combination therapy. Acta Trop 148: 97-104.

20. Mwingira $F$, Nkwengulila G, Schoepflin S, Sumari D, Beck H-P, Snounou G, Felger I, Olliaro P, Mugittu K, 2011. Plasmodium falciparum msp1, msp2 and glurp allele frequency and diversity in sub-Saharan Africa. Malar J 10: 79.

21. Branch OH, Takala S, Kariuki S, Nahlen BL, Kolczak M, Hawley W, Lal AA, 2001. Plasmodium falciparum genotypes, low complexity of infection, and resistance to subsequent malaria in participants in the Asembo Bay Cohort Project. Infec Immun 69: 7783-7792.

22. Coleman RE, Kumpitak C, Ponlawat A, Maneechai N, Phunkitchar V, Rachapaew N, Zollner G, Sattabongkot J, 2004. Infectivity of asymptomatic Plasmodium-infected human populations to Anopheles dirus mosquitoes in western Thailand. J Med Entomol 41: 201-208.

23. World Health Organization, 2017. WHO Malaria Policy Advisory Committee Meeting: Meeting Report. Geneva, Switzerland: WHO.

24. White NJ, 2017. The consequences of treating asymptomatic malaria parasitemia. Clin Infect Dis 64: 654-655.

25. Laishram DD, Sutton PL, Nanda N, Sharma VL, Sobti RC, Carlton $\mathrm{JM}$, Joshi H, 2012. The complexities of malaria disease manifestations with a focus on asymptomatic malaria. Malar J 11:29.

26. Cotter C, Sturrock HJ, Hsiang MS, Liu J, Phillips AA, Hwang J, Gueye CS, Fullman N, Gosling RD, Feachem RG, 2013. The changing epidemiology of malaria elimination: new strategies for new challenges. Lancet 382: 900-911. 